\section{Behandlung von Leukämien} Fortschritt durch gute präklinische und systematische klinische Forschung "made in Germany"

\section{Hallek ${ }^{1}$}

${ }^{1}$ Direktor der Klinik I für Innere Medizin, Universitätsklinikum Köln

Leukämien haben eine jährliche Inzidenz von etwa 13:100000 Einwohner. In Deutschland erkranken etwa 11000 Menschen pro Jahr. Die Erkenntnisse über der Leukämieforschung gehören zu den Glanzlichtern der medizinischen Forschung in den letzten Dekaden.

Ausgehend von Erkenntnissen zur chronischen myeloischen Leukämie (CML) wurde erstmals überhaupt verstanden, dass rekurrente Chromosomenveränderungen Krebs auslösen können. Dies führte später zur Identifizierung des krankmachenden Gens und Proteins BCR-ABL. Ein für diese Tyrosinkinase entwickelter Inhibitor, Imatinib, wurde zum ersten zielgerichteten, chemisch synthetisierten Medikament gegen Krebs und leitete eine neue Ära ein, die wir heute als „targeted therapies“ oder stratifizierte Medizin von Krebserkrankungen umschreiben [7]. Gemeinsames Charakteristikum dieses therapeutischen Ansatzes ist es, auf einem verbesserten pathogenetischen Verständnis basierende, gezielte Krebstherapien zu entwickeln, die in bestimmte Signalketten der Zelle eingreifen. Diese Entwicklung hat bei der CML ihren Ausgangspunkt genommen.

In den letzten 10 Jahren erlebt auch die Behandlung der chronischen lymphatischen Leukämie (CLL) einen sehr großen Aufschwung. Mehrere vor kurzem eingeführte, molekulare Therapeutika und monoklonale Antikörper haben die Therapie der Leukämie erheblich verbessert [2]. An erster Stelle zu nennen sind die Medikamente Rituximab, Obinutuzumab, Ibrutinib, Idelalisib und Venetoclax. Es ist zu erwarten, dass die CLL schon bald durch den kombinierten Einsatz von nicht zytotoxisch wirkenden Medikamenten kontrolliert werden kann [5].

Eine der CLL verwandte Erkrankung ist die Haarzellleukämie. Hier konnten vor kurzem molekulare Veränderungen im „Rapidly-accelerated-fibrosarcoma“(Raf)-Signalweg gezeigt werden, die übrigens der Veränderung beim malignen Melanom und anderen Erkrankungen sehr ähnlich sind. Ein dafür entwickelter Inhibitor wie Vemurafenib ist in der Lage, diese Erkrankung zurückzudrängen und stellt ein neues Therapieprinzip dar [3].

Bei den akuten Leukämien sind die Fortschritte schwieriger zu erzielen. Dennoch gehört die Forschung an der akuten lymphatischen Leukämie (ALL) des Erwachsenen ebenfalls zur Erfolgsgeschichte, da die deutsche Studiengruppe als eine der ersten, initial gefördert durch Mittel des Bundesforschungsministeriums, systematisch versucht hat, durch Kombination der wirksamen Chemotherapien eine Verbesserung der Lebenserwartung der Betroffenen zu erreichen. Dies ist der Studiengruppe nachhaltig gelungen [1]. In den letzten Jahren wurden darüber hinaus erfolgreich molekulare Therapien, aber auch Antikörper in die Therapiekonzepte eingefügt [6].

Die akute myeloische Leukämie (AML) zählt zu den schwierigsten Problemen in der Hämatologie und ist auch heute häufig nur durch eine Stammzelltransplantation heilbar [4]. Es gibt allerdings neue Ansätze, die basierend auf einer umfassenden molekularen Charakterisierung eine zunehmende Behandlung mit molekularen Therapeutika versuchen. Beispielsweise wird heute die akute Promyelozytenleukämie nicht mehr mit Chemotherapie, sondern durch die Gaben von Arsentrioxid und All-trans-Retinsäure (ATRA) therapiert. Zusammenfassend kann festgehalten werden, dass durch systematische präklinische und klinische Forschung zu molekularen Ursachen große therapeutische Fortschritte erzielt werden können. Die Forschung an Leukämien war und ist hierfür ein Schrittmacher, mit positiven Auswirkungen auf die gesamte Onkologie. Der Beitrag aus Deutschland zu dieser Entwicklung ist erfreulicherweise erheblich.

Interessenkonflikte: Research support: Roche, Gilead, Mundipharma, Janssen, Celgene, Pharmacyclics, AbbVie. Honoraria (speaker's bureau and/or advisory board): Roche, Gilead, Mundipharma, Janssen, Celgene, Pharmacyclics, AbbVie, Boehringer Ingelheim.

Literatur

1 Bassan R, Hoelzer D. Modern therapy of acute lymphoblastic leukemia. J Clin Oncol 2011; 29: $532-543$

2 Cramer P, Hallek M, Eichhorst B. State-of-the-Art Treatment and Novel Agents in Chronic Lymphocytic Leukemia. Oncol Res Treat 2016; 39: $25-32$

3 Dietrich S, Pircher A, Endris V et al. BRAF inhibition in hairy cell leukemia with low-dose vemurafenib. Blood 2016; 127: 2847-2855

4 Dohner H, Weisdorf DJ, Bloomfield CD. Acute myeloid leukemia. N Engl J Med 2015; 373: 1136-1152

5 Hallek M. Signaling the end of chronic lymphocytic leukemia: new frontline treatment strategies. Blood 2013; 122: 3723-3734

6 Hoelzer D. Targeted therapy with monoclonal antibodies in acute lymphoblastic leukemia. Curr Opin Oncol 2013; 25: 701 -706

7 Kantarjian H, Sawyers C, Hochhaus A et al. Hematologic and cytogenetic responses to imatinib mesylate in chronic myelogenous leukemia. N Engl J Med 2002; 346: 645-652

Bibliografie

DOI http://dx.doi.org/10.1055/s-0042-112404

Drug Res 2016; 66, Suppl. 1: S28-S28

(c) Georg Thieme Verlag KG Stuttgart · New York ·

ISSN 2194-9379

Korrespondenzadresse

Prof. Dr. med. Michael Hallek

Direktor der Klinik I für Innere Medizin

Universitätsklinikum Köln

Kerpener Straße 62

50937 Köln

michael.hallek@uni-koeln.de 